\title{
Transient elastography can be integrated into routine clinical practice for the evaluation of portal hypertension?
}

\author{
Beom Kyung Kim ${ }^{1,2}$ \\ 'Department of Internal Medicine, Yonsei University College of Medicine, Seoul; ${ }^{2}$ Institute of Gastroenterology, Yonsei University \\ College of Medicine, Seoul, Korea
}

Keywords: Transient elastography, Portal hypertension, Cirrhosis, Prediction

\section{See Article on Page 34}

Portal hypertension (PH) is a major consequence of liver tissue fibrogenesis in chronic liver disease (CLD).' During progression of $C L D$, the intrahepatic vasculature is remodeled and excess endogenous vasodilators are released, causing splanchnic arteriolar vasodilatation. Consequently, blood flow in the portal venous system would be increased, leading to PH. As the degree of PH becomes severe, it can result in complications such as the development of esophageal varices, variceal bleeding, ascites, spontaneous bacterial peritonitis and hepatorenal syndrome. ${ }^{2}$ Particularly in patients with decompensated cirrhosis, $\mathrm{PH}$ is responsible for significant morbidity and mortality. ${ }^{1-4}$ In this regard, precise assessment of PH allows accurate prediction of prognosis and is essential for managing CLD appropriately. Measurement of the hepatic venous pressure gradient (HVPG), the gradient between the wedged (i.e., balloon-occluded) hepatic venous pressure and the free hepatic venous pressure, has been accepted as the reference standard for assessing the degree of PH. Clinically significant PH (CSPH) defined as HVPG $\geq 10 \mathrm{mmHg}$, has been associated with formation of esophageal varices and poor prognosis. ${ }^{5-7}$ However, the routine use of this method in the clinical setting has been limited by its invasiveness and the need for expertise and specialized equipment such as angio-intervention unit. Thus, alternative approaches with acceptable diagnostic performance that allow clinicians to assess PH in patients with cirrhosis noninvasively have been needed.

Liver stiffness (LS) assessed using transient elastography (TE) was recently demonstrated to be a reliable and accurate noninvasive tool for assessing the degree of liver fibrosis. ${ }^{8,9}$ Recent largescale longitudinal studies also showed a significant association between $L S$ value and the risk of development of hepatocellular carcinoma ( $\mathrm{HCC}$ ) or cirrhotic complication in patients with chronic hepatitis B (CHB). 10,11 Theoretically, TE also reflects a progressive rise in portal pressure due to increased hepatic vascular resistance related to hepatic fibrosis. Accumulating evidence suggests that TE adequately reflects the findings of HVPG, indicating that it is a useful modality for evaluating PH and cirrhotic complications. ${ }^{12-18}$ TE has good performance for discriminating between patients with and without CSPH (area under the receiver operating characteristic curve [AUROC] 0.82-0.94)..$^{19}$ In addition, a TE value

\section{Abbreviations:}

CHB, Chronic hepatitis B; CLD, Chronic liver disease; CSPH, Clinically significant portal hypertension; HCC, Hepatocellular carcinoma; HVPG, Hepatic venous pressure gradient; LS, Liver stiffness; PH, Portal hypertension; TE, Transient elastography

\section{Corresponding author: Beom Kyung Kim}

Department of Internal Medicine, Yonsei University College of Medicine, 50-1 Yonsei-ro, Seodaemun-gu, Seoul 03722, Korea

Tel: +82-2-2228-1930, Fax: +82-2-393-6884

E-mail: beomkkim@yuhs.ac 
$<13 \mathrm{kPa}$ reliably excludes $\mathrm{CSPH}$, while values $>21 \mathrm{kPa}$ have an accuracy equal to that of HVPG $\geq 10 \mathrm{mmHg}$ for prediction of first clinical decompensation in patients with compensated cirrhosis. ${ }^{20}$ As variceal bleeding is the most important complication of $\mathrm{PH}$, the relationship between TE values and the presence of esophageal varices has been also investigated in several studies, ${ }^{13,21,22}$ which reported significant correlations between TE values and the presence of esophageal varices. Furthermore, Kim et al. ${ }^{23}$ conducted a systematic review and meta-analysis to identify the clinical usefulness of non-invasive TE for assessing PH as an alternative to HVPG in patients with CLD. The diagnostic accuracy of TE for CSPH was high (correlation $0.7480,95 \%$ confidence interval: $0.6464 ; 0.8236, P<0.0001)$. Furthermore, the sensitivity and specificity were 0.85 (range: $0.63-0.97$ ) and 0.71 (range: $0.50-0.93$ ), respectively; the AUROC was 0.88 (range: $0.76-0.99)$. Therefore, TE shows promise as a reliable and noninvasive procedure that should be integrated into clinical practice for the evaluation of $\mathrm{PH}$. In contrast, Llop et al. ${ }^{24}$ demonstrated a moderate correlation between TE and HVPG $(r=0.552)$ and patients with a TE value of 13.6-21 $\mathrm{kPa}$ had insufficient sensitivity and specificity to detect CSPH (HVPG $>10 \mathrm{mmHg}$ ). Thus, TE is not sufficiently accurate to replace HVPG due to its insufficient sensitivity or specificity. Furthermore, TE has limitations for clinical applications because of the wide range of cutoff values (ranges: $13.9-21.5 \mathrm{kPa}$ ) and variability in performance among studies (AUROC 0.76-0.85)..$^{13,21,22}$ Since PH is initiated by an increase in intrahepatic resistance, TE appropriately reflects mild-to-moderate $\mathrm{PH}$ or the initiation of $\mathrm{CSPH}$. However, the severity of $\mathrm{PH}$ is more dependent on the amount of portal blood inflow and peripheral hemodynamic changes than the stiffness of the hepatic parenchyma; thus, the predictive power of TE for PH might be limited. ${ }^{12}$ This explanation was also supported by the fact that the hemodynamic response to a non-selective $\beta$ blocker could not be accurately predicted using $\mathrm{TE} .{ }^{25}$

To overcome such shortcomings of TE alone in evaluating the $\mathrm{PH}$, several studies have shown that the combination of $\mathrm{TE}$, platelet count and spleen size by ultrasound has a superior diagnostic value for CSPH and esophageal varices than any of the three methods individually in patients with compensated cirrhosis of different etiologies. ${ }^{19} \mathrm{Kim}$ et al. ${ }^{26}$ recently proposed a novel prediction model, the LS-spleen diameter to platelet ratio score (LSPS); this uses TE values and the spleen diameter to platelet ratio, which reflect PH in patients with CHB. This model showed excellent diagnostic performance for the prediction of high-risk esophageal varices (AUROC 0.953; negative predictive value
94.7\%, positive predictive value $93.3 \%$ ). Another prospective study showed that LSPS was a reliable predictor of the development of variceal bleeding. CHB patients with an LSPS $\geq 5.5$ had a higher cumulative incidence rate of esophageal variceal bleeding during follow-up, and an LSPS score $\geq 6.5$ was an independent risk factor for variceal bleeding from high-risk esophageal varices, indicating that prophylactic treatment should be considered in these high-risk patients. ${ }^{10}$ However, these two studies had a limitation in that HVPG was not measured and that the correlation between HVPG and LSPS could not be evaluated. A recent validation study reported that $\geq 80 \%$ of patients were accurately classified using LSPS. Additionally, in this study, a modified LS-based score, (the varices risk score) was found to be superior to all other noninvasive tests for identifying patients with esophageal varices (AUROC 0.909); it classified $85 \%$ of patients correctly. ${ }^{18}$ The better performance of LSPS might be primarily due to the combination of different methods reflecting the various pathophysiological components of PH. Splenomegaly in cirrhotic patients is most likely the result of vascular disturbances, which are almost always due to greater portal pressure, whereas thrombocytopenia might be caused by either PH-induced splenic sequestration, or other mechanisms such as decreased thrombopoietin synthesis, shorter mean platelet lifetime, or myelotoxic effects of drugs or hepatitis viruses. $^{27-29}$

Although a systematic review and meta-analysis by Kim et al. ${ }^{23}$ showed the overall clinical usefulness of non-invasive TE for assessing PH as an alternative to HVPG in patients with CLD, that study had potential limitations. First, only eight studies were included, so the robustness of the conclusions in terms of the evaluation of the usefulness and performance of TE might be limited. Second, the characteristics of the included studies, such as patient characteristics, etiologies of cirrhosis and diagnostic thresholds, were various. Third, it included only studies published in English, potentially leading to a language bias.

In conclusion, based on the positive results of TE for assessing $\mathrm{PH}, \mathrm{TE}$ can be integrated into routine clinical practice for the evaluation of PH. However, LS itself might not be sufficient to estimate and replace the HVPG accurately; therefore, complementary methods are required.

\section{Conflicts of Interest}

The author has no conflicts to disclose. 


\section{REFERENCES}

1. Kim MY, Cho MY, Baik SK, Park HJ, Jeon HK, Im CK, et al. Histological subclassification of cirrhosis using the Laennec fibrosis scoring system correlates with clinical stage and grade of portal hypertension. J Hepatol 2011;55:1004-1009.

2. Jang YO, Jun BG2 Baik SK, Kim MY, Kwon SO. Inhibition of hepatic stellate cells by bone marrow-derived mesenchymal stem cells in hepatic fibrosis. Clin Mol Hepatol 2015;21:141-149.

3. Eom YW, Shim KY, Baik SK. Mesenchymal stem cell therapy for liver fibrosis. Korean J Intern Med 2015;30:580-589.

4. Eom YW, Kim G, Baik SK. Mesenchymal stem cell therapy for cirrhosis: Present and future perspectives. World J Gastroenterol 2015;21:10253-10261.

5. Baik SK. Haemodynamic evaluation by Doppler ultrasonography in patients with portal hypertension: a review. Liver Int 2010;30:14031413.

6. Kim MY, Suk KT, Baik SK, Kim HA, Kim YJ, Cha SH, et al. Hepatic vein arrival time as assessed by contrast-enhanced ultrasonography is useful for the assessment of portal hypertension in compensated cirrhosis. Hepatology 2012;56:1053-1062.

7. Kim MY, Jeong WK, Baik SK. Invasive and non-invasive diagnosis of cirrhosis and portal hypertension. World J Gastroenterol 2014;20:4300-4315.

8. Castera L. Noninvasive assessment of liver fibrosis. Dig Dis 2015;33:498-503.

9. Kim DY, Kim SU, Ahn SH, Park JY, Lee JM, Park YN, et al. Usefulness of FibroScan for detection of early compensated liver cirrhosis in chronic hepatitis B. Dig Dis Sci 2009;54:1758-1763.

10. Kim BK, Kim DY, Han KH, Park JY, Kim JK, Paik YH, et al. Risk assessment of esophageal variceal bleeding in B-viral liver cirrhosis by a liver stiffness measurement-based model. Am J Gastroenterol 2011;106:1654-1662, 1730.

11. Jung KS, Kim SU, Ahn SH, Park YN, Kim D Y, Park JY, et al. Risk assessment of hepatitis B virus-related hepatocellular carcinoma development using liver stiffness measurement (FibroScan). Hepatology 2011;53:885-894.

12. Vizzutti F, Arena U, Romanelli RG, Rega L, Foschi M, Colagrande $S$, et al. Liver stiffness measurement predicts severe portal hypertension in patients with HCV-related cirrhosis. Hepatology 2007:45:1290-1297.

13. Lemoine M, Katsahian S, Ziol M, Nahon P, Ganne-Carrie N, Kazemi $F$, et al. Liver stiffness measurement as a predictive tool of clinically significant portal hypertension in patients with compensated hepatitis C virus or alcohol-related cirrhosis. Aliment Pharmacol Ther 2008;28:1102-1110.

14. Bureau C, Metivier S, Peron JM, Selves J, Robic MA, Gourraud PA, et al. Transient elastography accurately predicts presence of significant portal hypertension in patients with chronic liver disease. Aliment Pharmacol Ther 2008;27:1261-1268.

15. Sánchez-Conde M, Miralles P, Bellón JM, Rincón D, Ramírez M, Gutiérrez I, et al. Use of transient elastography (FibroScan $®$ ) for the noninvasive assessment of portal hypertension in HIV/HCV-coinfected patients. J Viral Hepat 2011;18:685-691.

16. Carrión JA, Navasa M, Bosch J, Bruguera M, Gilabert R, Forns X. Transient elastography for diagnosis of advanced fibrosis and portal hypertension in patients with hepatitis $\mathrm{C}$ recurrence after liver transplantation. Liver Transpl 2006;12:1791-1798.

17. Reiberger $T$, Ferlitsch A, Payer BA, Pinter M, Schwabl P, Stift J, et al. Noninvasive screening for liver fibrosis and portal hypertension by transient elastography--a large single center experience. Wien Klin Wochenschr 2012;124:395-402.

18. Berzigotti A, Seijo S, Arena U, Abraldes JG, Vizzutti F, García-Pagán $J C$, et al. Elastography, spleen size, and platelet count identify portal hypertension in patients with compensated cirrhosis. Gastroenterology 2013;144:102-111.

19. Castera L, Pinzani M, Bosch J. Non invasive evaluation of portal hypertension using transient elastography. J Hepatol 2012;56:696703.

20. Robic MA, Procopet B, Métivier S, Péron JM, Selves J, Vinel JP, et al. Liver stiffness accurately predicts portal hypertension related complications in patients with chronic liver disease: a prospective study. J Hepatol 2011;55:1017-1024.

21. Castéra L, Le Bail B, Roudot-Thoraval F, Bernard PH, Foucher J, Merrouche $\mathrm{W}$, et al. Early detection in routine clinical practice of cirrhosis and oesophageal varices in chronic hepatitis C: comparison of transient elastography (FibroScan) with standard laboratory tests and non-invasive scores. J Hepatol 2009;50:59-68.

22. Kazemi F, Kettaneh A, N'kontchou G, Pinto E, Ganne-Carrie N, Trinchet JC, et al. Liver stiffness measurement selects patients with cirrhosis at risk of bearing large oesophageal varices. J Hepatol 2006:45:230-235.

23. Kim G, Kim MY, Baik SK. Transient elastography versus hepatic venous pressure gradient for diagnosing portal hypertension: A systematic review and meta-analysis. Clin Mol Hepatol 2017;23:34-41.

24. Llop E, Berzigotti A, Reig M, Erice E, Reverter E, Seijo S, et al. Assessment of portal hypertension by transient elastography in patients with compensated cirrhosis and potentially resectable liver tumors. J Hepatol 2012;56:103-108.

25. Reiberger T, Ferlitsch A, Payer B A, Pinter M, Homoncik M, PeckRadosavljevic M. Non-selective beta-blockers improve the correlation of liver stiffness and portal pressure in advanced cirrhosis. J Gastroenterol 2012;47:561-568.

26. Kim BK, Han KH, Park JY, Ahn SH, Kim JK, Paik YH, et al. A liver stiffness measurement-based, noninvasive prediction model for high-risk esophageal varices in B-viral liver cirrhosis. Am J Gastroen- 
terol 2010;105:1382-1390.

27. Giannini E, Botta F, Borro P, Risso D, Romagnoli P, Fasoli A, et al. Platelet count/spleen diameter ratio: proposal and validation of a non-invasive parameter to predict the presence of oesophageal varices in patients with liver cirrhosis. Gut 2003;52:1200-1205.

28. Giannini EG. Review article: thrombocytopenia in chronic liver dis- ease and pharmacologic treatment options. Aliment Pharmacol Ther 2006;23:1055-1065.

29. Thabut D, Ratziu V, Trabut JB, Poynard T. Prediction of oesophageal varices with platelet count/spleen diameter ratio or platelets alone. Gut 2004;53:913-914. 